\title{
Design of MMC Hardware-in-the-Loop Platform and Controller Test Scheme
}

\author{
Guoqing Li, Di Zhang, Yechun Xin, Shouqi Jiang, Weiru Wang, and Jiahui Du
}

\begin{abstract}
The hardware-in-the-loop (HIL) simulation is an effective method to verify the overall function of the flexible HVDC transmission control and protection device. With this method, debugging the control and protection device can make the system run safely and stably after being put into operation. Therefore, a hardware-in-the-loop simulation platform modular multilevel converter (MMC) based on RT-LAB is established in this paper. Data merging between a converter valve control system and the real-time simulator is realized by high-speed optical fiber communication protocol conversion chassis, and the high-speed communication interface is designed to meet the requirements of the communication rate. Aiming at the control performance of the physical device, the test scheme is designed, and the test methods of voltage balance control and circulating current suppression are proposed. The closed-loop test of control and protection device is carried out by the active power step and AC/DC fault test. The above test verifies the validity of the HIL simulation platform of the MMC and the rationality of the testing scheme, and can meet the performance testing requirements of the control and protection device.
\end{abstract}

Index Terms - Control and protection device, device debugging, hardware-in-the-loop (HIL), modutar miltilevel converter (MMC), simulation test.

\section{INTRODUCTION}

$\mathrm{N}_{\mathrm{b}}^{\mathrm{o}}$ OWADAYS, building up of a global energy ntework has become an international consensus. It is urgent to innovate the advanced transmission methods to develop long distance and large capacity transmission, and to improve not only the efficiency of transmission and the utilization of resources, but also the security, flexibility, and controllability of the power grid. Flexible high voltage direct current (HVDC) transmission has an independent, accurate, flexible and convenient active/ reactive power control mode, and it has the advantages of rapid recovery and control after power grid fault, which has a wide application prospect [1]-[4]. The modular multilevel converter (MMC) has become a trend in the future, because of its outstanding advantages, such as high output waveform quality and minimal loss. Compared with the traditional DC

Manuscript received June 21, 2018. This work was supported in part by the National Key Research and Development Program of China (2016YFB0900903), and National Natural Science Foundation of China (51607032).

All authors are with the College of Electrical Engineering, Northeast Electric Power University, Jilin, 132012, China. (e-mail: 923114180@qq.com)

Digital Object Identifier 10.24295/CPSSTPEA.2019.00014 commutation valve, two-level, and three-level converter valves, the modules of the modular multilevel converter need to be controlled independently. The complex structure of the control system also brings new problems to the design and test of the control system.

In order to ensure the reliable operation of the flexible HVDC converter valve, the domestic and foreign scholars and the research institutes have conducted a lot of research work on the design and simulation tests of the flexible HVDC transmission control system. In recent years, the modular multilevel converter real-time digital simulation of MMC based on FPGA (Field-Programmable Gate Array) technology has been greatly developed, because of the difficulties in the electromagnetic transient simulation of the switch valve caused by a large number of switching devices. The FPGA real-time digital simulation system and the control system are combined with the hardware-in-the-loop simulation which provides a means of improve the performance testing of the control system [5]-[6]. At the same time, the appropriate equivalent model is conducive to the simulation analysis of the system. For modular multilevel converter topology, the following three methods are usually used for steady-state analysis. The IGBT and its antiseries diode in the sub-module are equivalent to one switch to improve the simulation speed [7]; MMC averagely simplified model method [8]; the capacitor in the sub-module is replaced by the time-domain Thevenin equivalent branch, and then the sub-module is equivalent to a Thevenin equivalent branch. Finally, the sub-module Thevenin equivalent branch is cascaded to form the Thevenin equivalent branch of the whole bridge arm [9]. The efficiency of steady-state simulation analysis is greatly improved by the methods above, but there are still some limitations in the system simulation with blocking sub-modules. In order to better simulate the dynamic response process of $\mathrm{MMC}$, an equivalent sub-module is used to replace an arm, which is equivalent to a Thevenin equivalent circuit in the case of deblocking MMC, while the arm is equivalent to a halfbridge uncontrolled sub-module with lumped parameters in the case of MMC blocking [10].

The control and protection system is the "brain" of the whole flexible HVDC transmission project. In the field of control and protection testing, MMC has a large number of sub-modules. Owing to the complexity of the control system, the system test is difficult and the workload is large before the actual project is put into operation. In order to simulate and debug the control and protection devices, it is an effective means of testing and de 
bugging all kinds of steady-state, transient and fault conditions of the controller using the HIL simulation method [11]-[12]. The HIL simulation test can verify the real-time performance of the controller and the correctness of the protection device action under conditions close to the actual operating conditions, and modify the relevant settings of the actual controller. The HIL simulation is a relatively mature test method and has been widely used in the design verification of control platforms [13]-[15].

Most of the existing hardware-in-the-loop simulation methods introduced in the literature are still aimed at the verification of the designed controller, and few of them are fully tested and validated for the whole system. However, how to realize the communication interface between the digital simulation system and the actual device has not been referenced [16]-[17]. Therefore, in order to test the MMC flexible HVDC transmission control protection device, this paper designs a hardware-in-theloop simulation test system based on RT-LAB. The MMC main circuit using the Thevenin equivalent model of reference [10] runs in RT-LAB. The data merge is realized through a highspeed fiber-optic communication protocol conversion chassis. The physical side accesses control protection devices and forms a closed loop. According to the performance of the control protection device, the test scheme of converter station control and valve control is designed, and the dynamic performance test and function test of the actual control and protection device are completed by using the built platform. Unlike the traditional method, this method can reduce the actual project site debugging process and shorten the development cycle of the control and protection system.

\section{Flexible DC System Structure}

The overall structure and control system structure of the MMC flexible HVDC transmission system is given in Fig. 1(a) and (b). The control system is mainly composed of inner loop current controller, outer loop controller, phase locked loop, and pulse generation link. The two-terminal of the converter station needs to control the active component and the reactive component. Under normal circumstances, for a two-terminal active AC system, the rectifier side is generally used to control the active power and reactive power, and the inverter side controls the DC side voltage and reactive power. The converter valve control system receives the voltage reference wave from the converter station control and modulates the voltage using the nearest level modulation (NLM) or pulse width modulation (PWM) [18][19]. The trigger signal is applied to the sub-module to complete the switching of the state so that the output voltage waveform is close to a sine wave. The converter valve control system also needs to complete the control of the sub-module capacitance voltage balance.

The safe and stable operation of the equipment in the flexible HVDC transmission system is the main responsibility of the protection system. In the event of a fault or abnormality, the faulty or abnormal operating equipment is quickly removed from the system to prevent equipment damage or other normal working parts from being affected so that it can ensure the safe operation of the DC system. This ensures the safe operation

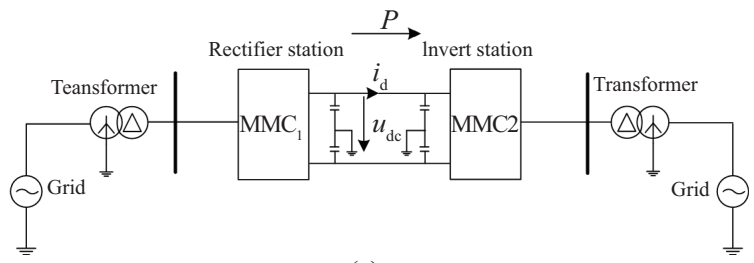

(a)

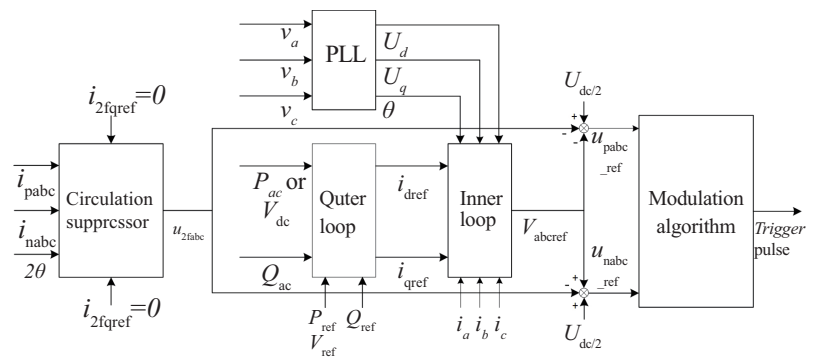

(b)

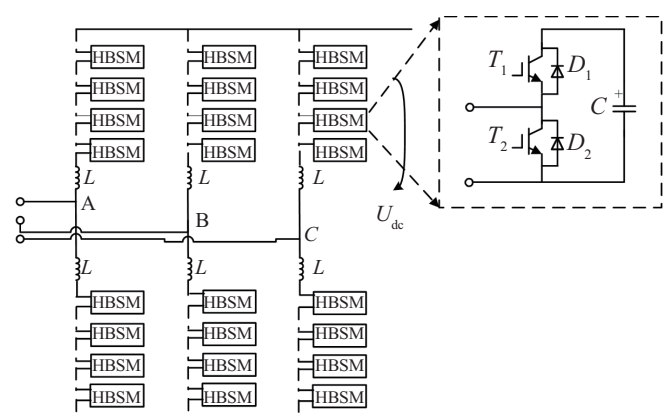

Fig. 1. MMC-HVDC system and control structure. (a) MMC-HVDC system. (b) Control system. (c) Equivalent model.

of the DC system. According to the different protected areas, protection can be generally divided into $\mathrm{AC}$ area protection [20] [21], converter area protection, and DC area protection [22].

Due to the high controllability of the MMC-HVDC, the control and protection system largely determines its dynamic performance. Therefore, it is necessary to carry out simulation tests on the converter station control, converter valve control and protection devices by the HIL simulation platform to test whether the performance can fulfill operational requirements.

\section{Simulation Platform Design}

\section{A. Real-Time Simulator}

The characteristics and safety requirements of the power system determine that the exploration and research of the actual power grid greatly depend on simulation. Due to the high precision of the switching action of the power electronic devices in the flexible HVDC transmission, the simulation step size used in the simulation analysis is generally about $100 \mu \mathrm{s}$ in order to accurately simulate and analyze the switching behavior of the device. Therefore, electromagnetic transient simulation tools are mainly used in the research of flexible HVDC transmission, such as MATLAB/Simulink in RT-LAB. 


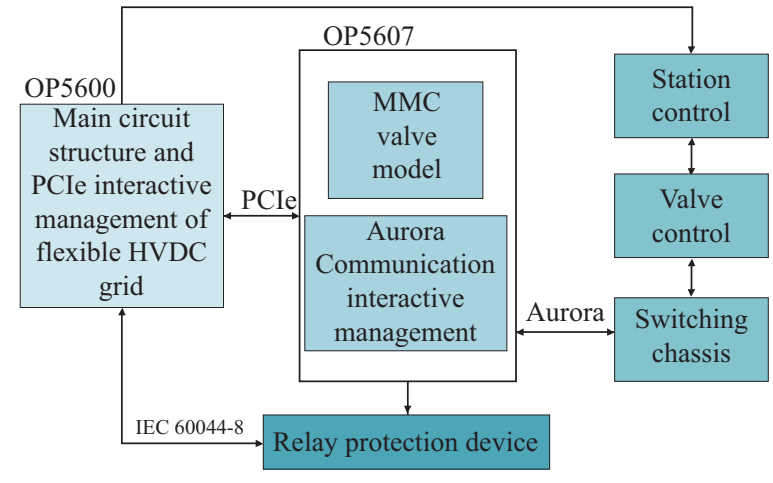

Fig. 2. System interface device overall structure.

The implementation of the MMC main circuit depends on the upper computer and the target machine. The upper computer completes tasks, such as modeling, on-line parameter adjusting, signal monitoring, and $\mathrm{C}$ code generating to download the target machine. The target computer adopts the OP5600 with the Redhat operating system and the FPGA-based OP5607 as the realtime simulator for the HIL simulation applications.

By using the RT-LAB software running on the upper computer to achieve the connection between the upper computer and the target computer, RT-LAB can divide the complex model into multiple parallel subsystems and then allocate it to different CPU cores of the OP5600. The first is to build the system in the MATLAB/ Simulink environment. Then, according to the principle of model segmentation under RT-LAB, a series of operations such as model compilation, interface definition, and loading code are completed. The digital model is then converted to real-time code running on the target machine. Finally, the target machine will output all the signals.

The HIL simulation experiment platform designed in this paper completes the construction of the MMC main circuit on the upper computer. The real-time monitoring of the running state of the converter station is completed by the computer, and its functions are verified.

\section{B. Overall System Architecture}

Fig. 2 shows the overall system architecture of the interface devices. The OP5600 in the diagram is mainly used as the main circuit calculation. The main circuit components and the corresponding parameter settings are calculated in the CPU of the OP5600. OP5607 is mainly used for converter valve simulation and IO interface, which It receives the drive signal through the optical fiber and sends out the corresponding submodule voltage. The IO interface is mainly used for the analog output and the corresponding digital signal input and output. The converter valve control device converts multi-channel optical signals into one or more signals by means of a communication protocol conversion chassis, completes the data integration work, and connects the OP5607 through the Aurora protocol to achieve information exchange including switching commands, capacitor voltage, and bridge arm current. The OP5607 internally completes part of the functional simulation of the sub-modules,

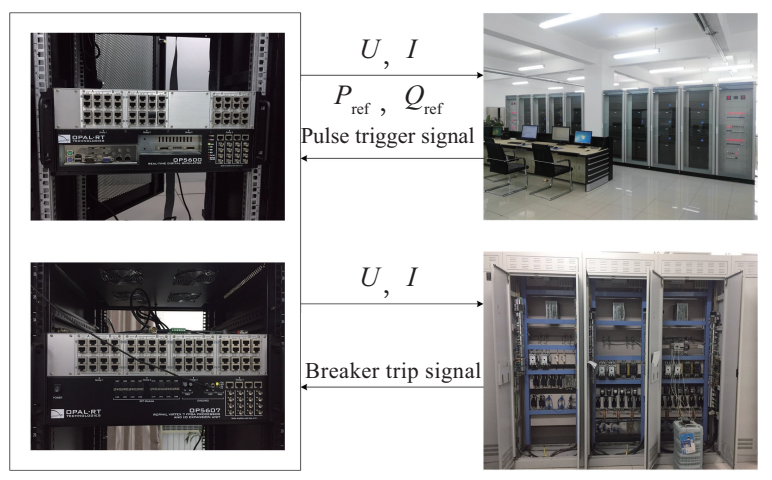

Fig. 3. Diagram of hardware-in-the-loop simulation platform hardware connection.

and the data of the capacitance voltage of the sub-module is transmitted to the OP5600 via PCIe. The human-computer interaction interface is set in the monitoring background to realize the sub-module state, the function of the sub-module, and the display and setting of the control target and instruction. The converter station control device and the relay protection device are respectively connected with the OP5600, OP5607 through the IEC60044-8 protocol.

Fig. 3 shows the hardware connection diagram of the HIL simulation platform. The converter station control device needs to collect voltage signals on the $\mathrm{AC}$ side to complete the phase locked loop control. It uses the received DC voltage, active and reactive power reference values, and the voltage reference waves of the six bridge arms which are calculated through the inner and outer loop control. After electro-optical conversion, it is transmitted from the optical fiber to the converter valve control. The converter valve control passes through the photoelectric conversion and will receive the reference wave generated by the converter station control, adopting the nearest level modulation method to determine the number of sub-module inputs. It then sorts each sub-module according to the capacitor voltage based on the current direction of the bridge arm (positive or negative) that the sub-module with low or high capacitor voltage should be charged or discharged to generate the trigger signal of the sub-module. This completes the sub-modules' voltage balance control. The converter valve control also needs to collect the bridge arm current to complete the suppression of the interphase circulating current of the converter. The protection device needs to collect the DC-side signals in addition to the above-mentioned voltage and current signals to determine whether a protection action is triggered when a fault occurs and send the protection action signal and breaker trip signal back to the RT-LAB. In this way, a closed loop system is formed, which can accurately reflect the characteristics of the flexible HVDC transmission system and can better test the control and protection device.

\section{Data Merge}

Because of the large number of sub-modules and the need for the digital simulator to send the capacitor voltage and operating status of the sub-module to the converter valve control device, and to receive the trigger command, RT-LAB has a one-to-one 


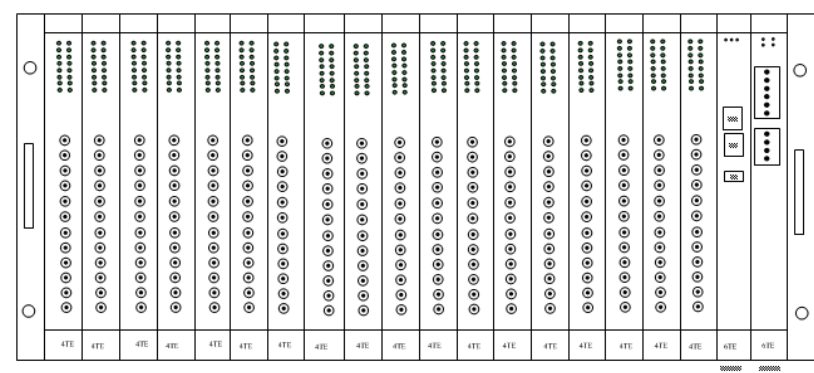

Fig. 4. Protocol conversion control box.

correspondence with the converter valve control interface. For the 300 sub-module capacitance voltages (without considering redundancy) of the single converter valve, this is complicated to operate. For this reason, the protocol conversion control chassis adopts the Aurora communication protocol, which is connected the valve base controller (VBC) device with through the Small Form-factor Pluggables (SFP) optical fiber module. All of the communication optical fibers between the VBC and the MMC sub-module digital model need to be connected to the protocol conversion control chassis. The protocol conversion control chassis assumes the task of connecting the entire simulation system. Each fiber interface board has six pairs of fiber interfaces and has a one-to-one correspondence with the VBC interface, and the structure of the interface board has a similar structure to the bridge control board of the VBC. The multimode optical fiber serves as a communication medium. The optical transmitter uses the HFBR-1414 optical transmitter module and the optical receiver uses the HFBR-2412 optical receiver module. A fullduplex information exchange channel is formed by the VBC and each sub-module via two optical fiber lines.

The protocol conversion control box Shown in Fig. 4 is responsible for the task of the whole simulation system. The standard 6U19 inch cabinet chassis design is adopted, it has 18 fiber interface cards, 1 main control board, 1 power board, and 1 back board. As shown in Fig. 5 for a data merge method, the real-time simulator OP5607 connects with the SFP light module on the FPGA board in the protocol conversion control chassis through the ultra-high-speed optical fiber. The communication protocol uses Aurora $8 \mathrm{~B} / 10 \mathrm{~B}$, bandwidth $5 \mathrm{GHz}$. It can be connected with the valve control system through FC7161 optical signal processing board. The protocol adopts IEC60044-8, which realizes the data merge between the multi-channel optical fiber data of the converter valve control system and the OP5607 realtime simulator, thus completing the HIL simulation of the MMC system. The design of the high-speed communication interface adopts SFP fiber with $2.5 \mathrm{Gbps}$ bandwidth and AURORA protocol to complete the communication. The optical signal processing board is connected to the valve control system, and the data is encapsulated in $8 \mathrm{~B} / 10 \mathrm{~B}$. The data transmission of $8 \mathrm{~K}$ can be completed in $2 \mu \mathrm{s}$. The protocol conversion control chassis uses IEC60044-8 protocol to communicate with VBC. The interface is set to 32 bits. It takes four microseconds to complete a full data transmission. The FPGA uses a $200 \mathrm{MHz}$ clock parallel operation. If the 300 sets of data are completely reorganized, it

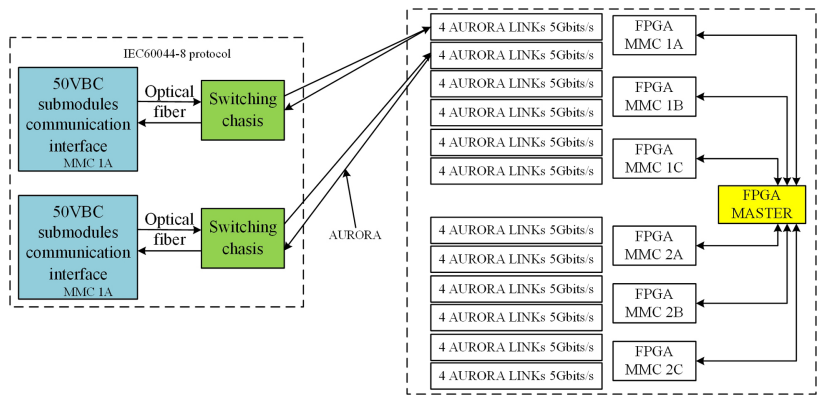

Fig. 5. Data merging method.

needs $2.5 \mu$ s which is the data transmission time plus the data reorganization time (up to $6.5 \mu \mathrm{s}$ ). In addition to data transmission time and data reorganization time, there is also backplane transmission delay in the process of data transmission. Each optical fiber interface board is responsible for communicating with $\mathrm{VBC}$, and the data received by the optical fiber interface board needs to be transmitted to the main control board through the backplane bus. The backplane uses high-speed LVDS pointto-point data transmission, and the transmission time of 32 bit data is about $6.25 \times 10^{-6} \mathrm{~s}$. Therefore, the backplane transmission delay can be ignored in the communication rate calculation. The highspeed communication interface should be able to complete the transmission of 300 sub-module capacitor voltages in $10 \mu$ s step.

\section{Design of the Device Test Method}

The test of the control and protection device for the flexible DC transmission system can be divided into a functional test and a dynamic performance test. The purpose of the functional test is to verify, optimize and test the overall functions of the complete control and protection equipment, including the verification of the accuracy of the design of the control and protection software, the test of the mutual cooperation between the control and protection equipment along with its functions under various operating modes as well as the accuracy of the interaction between the $\mathrm{AC}$ and $\mathrm{DC}$ system, and the verification of the correctness of sequence control logic and operation procedures.

The dynamic performance test mainly tests the transient characteristics of the flexible DC transmission system, checks the interaction between the control and protection device and the $\mathrm{AC} / \mathrm{DC}$ system, and selects and verifies the control protection parameters. It also optimizes the response of the complete control equipment under various operating conditions and checks whether the protection configured in each area is reliable. The tests for the performance of the control device include sequential control, converter charging, open line test, deblock/block test, control mode switching, reactive power control, voltage balance control, circulation suppression, power lift, power step, DC voltage step, etc. In order to verify the reliable operation of the protection device, it is necessary to test AC bus fault, valve fault, and DC polar fault.

According to the control function of the physical device, on the basis of completing the equipment interface signal, 
the internal communication, and the trigger pulse test, the performance test of the converter station control should be completed first. After the station control performance is accurate, the performance of the converter valve control is verified, and the steady performance test is carried out.

The converter station control performance test is divided into six tests. 1) The purpose of the converter charging test is to verify the accuracy of the valve trigger phase sequence and the control protection system phase-locked link. The sub-module charge is divided into two processes of uncontrolled rectifier charge and controllable charge, in which the controlled charge is completed under the control of the constant DC voltage. 2) The open line test (OLT) test is the deblocking test for the first active state of the converter valve. The purpose is to check whether the control system can correctly control the $\mathrm{DC}$ voltage and test the insulation of the main equipment. In order to ensure that the DC voltage does not exceed the maximum limit, simply set the DC voltage target value and voltage change rate and monitor the actual value of the DC voltage. 3) The sequential deblocking test provides insight as to whether the DC bus voltage and the system operation is stable after the uncontrolled rectifier charging is completed and the charging resistor exits to the end of the inverter deblocking process. 4) Control mode switching is when the converter station is converted from PQ to PV (or PV switching to PQ). It is observed whether the voltage and current waveform are smooth, whether there is a large fluctuation, and whether the system can maintain stable operation. 5) During the power (voltage) step, it is tested whether the system can respond quickly and complete the accurate tracking of the target value for the given power (voltage) instruction value. 6) STATCOM verifies the performance of the static var compensator and checks the current second harmonic frequency of the control protection system current.

As the core element in the secondary system of HVDC transmission, the converter valve control device is the interface device which connects the converter valve and the DC control system. It is an important part to control and protect the converter valve. Converter valve control is mainly responsible for pulse generation, voltage balance control, and circulation suppression.

The converter valve control performance test can be divided into three different tests, including voltage balance control, circulation suppression, and the protection function. 1) Voltage balance control keeps the deviation between the capacitor voltage and the calculated value of the sub-module within the target value. 2) During the circulating current suppressing test, the bridge arm current is monitored, and Fourier decomposition is performed to observe the proportion of the secondary harmonic negative sequence component. 3) When a fault occurs inside the valve or an over-current or over-voltage occurs in the bridge arm, the protection function checks the requested trip command to see whether it can be sent to the upper computer in time.

Because there are too many test projects mentioned above, this paper only designs two test methods to verify the control function of the voltage balance control and circulating current suppression control in the valve control test. The test flow is introduced as follows.

Fig. 6 is a flowchart for the test of voltage balance control.

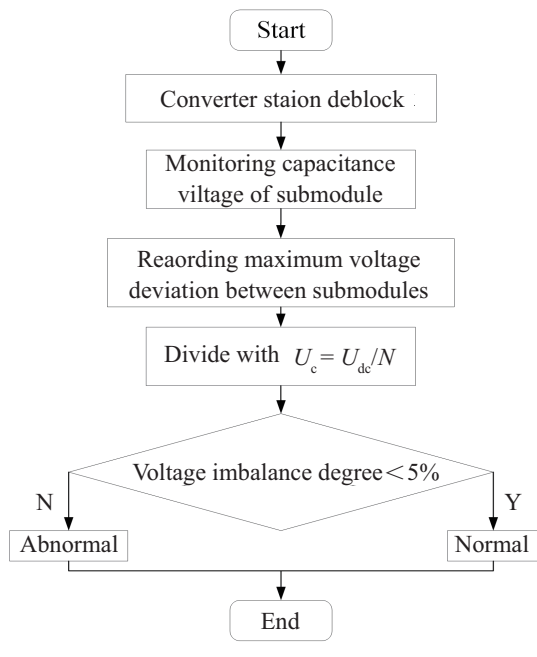

Fig. 6. The test flowchart of voltage sharing control.

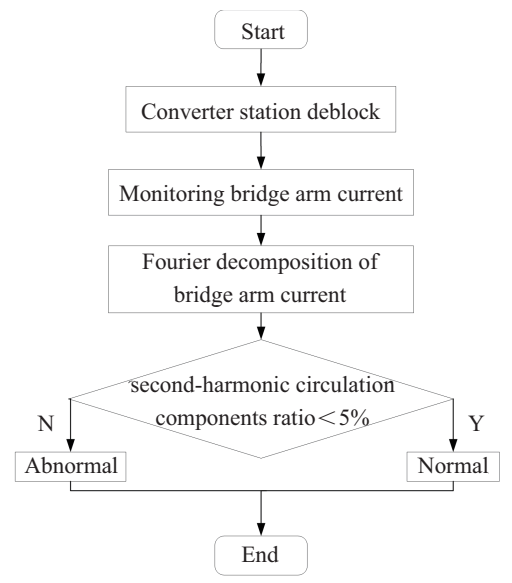

Fig. 7. The test flowchart of circulation suppression.

When the converter is unlocked, the voltage balance control begins to be put in. The capacitance voltage of each module is monitored and records the ratio of the maximum deviation value of each capacitance voltage to $U_{\mathrm{c}}=U_{\mathrm{dc}} / N$. If the voltage unbalance is less than $5 \%$, the effect of voltage sharing control is better.

Fig. 7 shows the circulating current suppression test flow chart. It monitors the current of each phase of the bridge arm in the converter valve, decomposes the bridge arm current by Fourier decomposition, and observes the content of the secondary harmonic component. If the circulation component is less than $5 \%$, the control effect of the circulation suppression is proved to be normal.

\section{Simulation Verification}

In order to verify the performance and testability of the built simulation platform, multiple tests were conducted under steady state, dynamic and fault conditions respectively. A two-terminal active system with a 51-level MMC-HVDC is running in the real-time simulator RT-LAB. The MMC modulates using the nearest level modulation. Station 1 adopts the control method of constant active power and reactive power; station 2 adopts 
TABLE I

Parameters of Simulation Test System

\begin{tabular}{lc}
\hline \hline AC voltage & $230 \mathrm{kV}$ \\
AC frequency & $50 \mathrm{~Hz}$ \\
DC voltage & $\pm 160 \mathrm{kV}$ \\
Converter rated capacity & $500 \mathrm{MVA}$ \\
Bridge arm inductor & $28 \mathrm{mH}$ \\
Number of sub-modules & 50 \\
Submodule capacitance & $5 \mathrm{mF}$ \\
DC capacitance & $2 \mu \mathrm{F}$ \\
\hline \hline
\end{tabular}

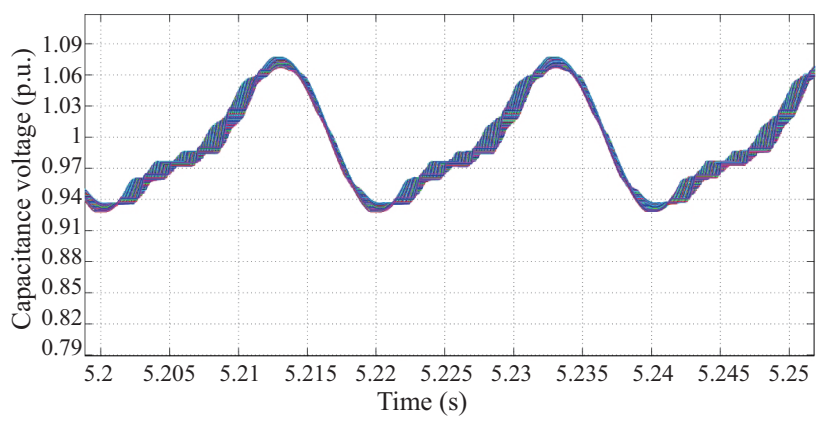

Fig. 8. Waveforms of voltage sharing control test.

the control method of constant DC voltage and reactive power, of which, $P=-500 \mathrm{MW}$ and $Q=100 \mathrm{MVar}$. The converter transformers connections are connected using $\mathrm{Y}_{\mathrm{N}} \mathrm{d}$. The main circuit related parameters are shown in Table I.

This article will mainly analyze the test contents of voltage balance control test, circulation suppression test, power step test, and $\mathrm{AC} / \mathrm{DC}$ side fault (converter valve side single-phase grounding and DC bipolar short-circuit).

\section{A. Voltage Balance Control Test}

Fig. 8 shows the results of the voltage balance control test waveform. As previously mentioned, it can be determined that the voltage balance control of the sub-module capacitance voltage is performed well after the converter station is deblocked and voltage balance control is applied. The voltage fluctuation is small, and the maximum deviation of the capacitor voltage imbalance is not more than $3 \%$. This is less than the specified $5 \%$, which verifies that the converter valve control has a normal effect on the capacitor voltage balance control.

\section{B. Circulation Suppression Test}

In order to verify the suppressing performance of the VBC to the interphase circulating current of the bridge arm, the circulating current suppression control test is carried out.

Fig. 9 shows the current and circulating current of the upper and lower arm of phase A, the waveform of the grid side current and the harmonic content. The current has no obvious distortion and the waveform quality is better. The secondary harmonic content in the bridge arm current is less than $2 \%$, which is far below $5 \%$, indicating that the converter valve control has an

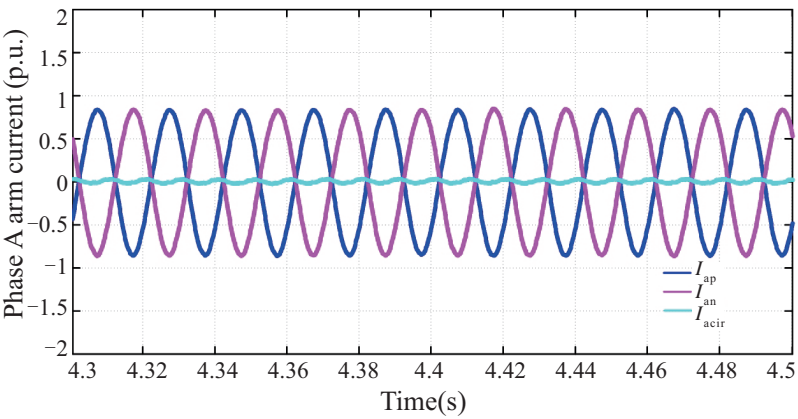

(a)

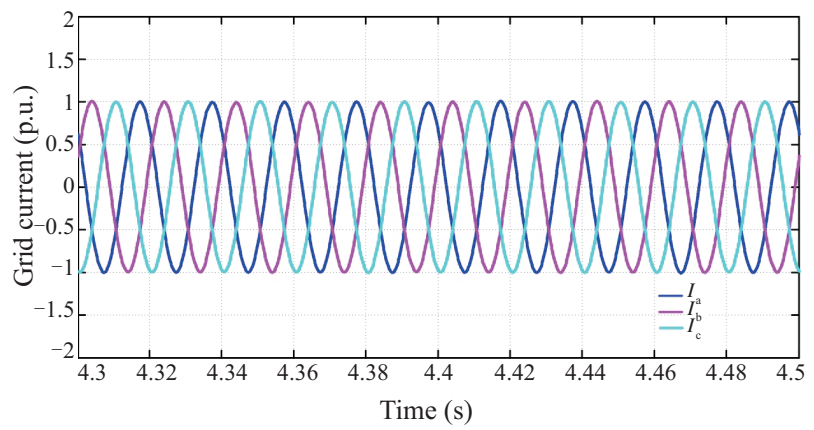

(b)

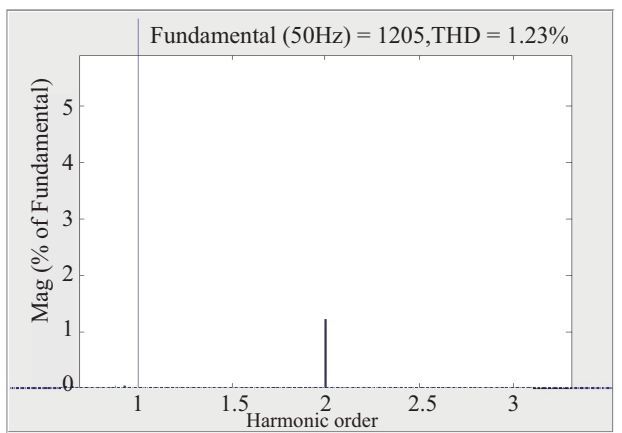

(c)

Fig. 9. Circulation suppression waveforms. (a) Phase A arm current. (b) AC current. (c) Harmonic content waveforms.

obvious effect on suppressing the phase-to-phase circulation of the bridge arm, and the control performance is good.

\section{C.Power Step Test}

In order to verify the dynamic performance of the platform, the active power test was carried out. Active power is reversed from $-500 \mathrm{MW}$ to $300 \mathrm{MW}$ at $5 \mathrm{~s}$, and reactive power is maintained at 100 Mvar. Fig. 10 shows the waveform of power, phase $\mathrm{A}$ arm current and capacitance voltage.

According to Fig. 10, the active power step is rapidly completed and the tracking is accurate. Reactive power is subject to a certain disturbance, but within $0.3 \mathrm{~s}$ the fixed value is restored. The internal current $I_{\text {acir }}$ of phase A is fluctuated greatly during the transition process. It can transit smoothly under the action of the circulating current suppressor. The amplitude of AC component of arm current $I_{\text {ap }}$ and $I_{\text {an }}$ is decreased, and there is a more obvious distortion in the transition process. The capacitance voltage is fluctuated slightly after reversing, but it 


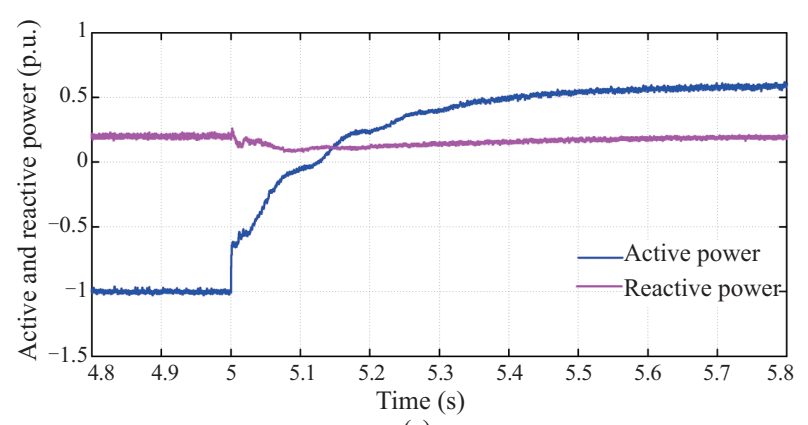

(a)

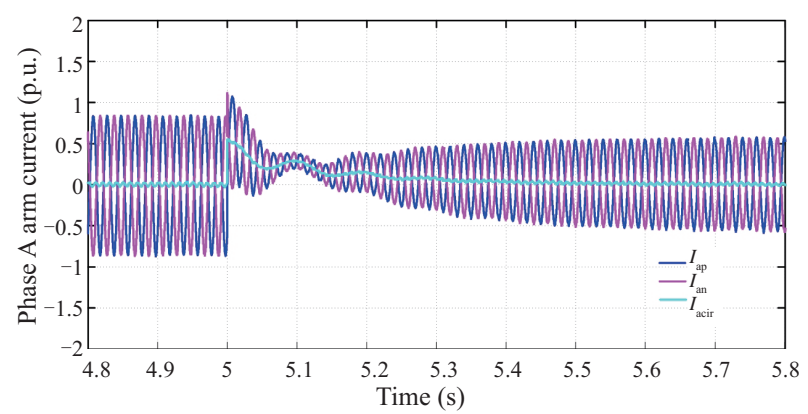

(b)

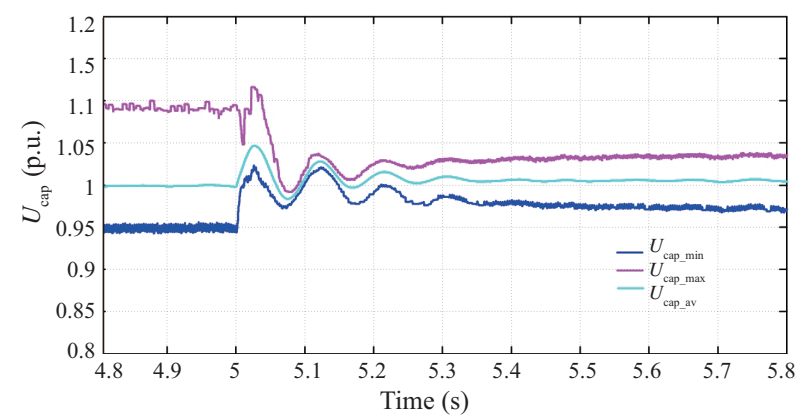

(c)

Fig. 10. Waveforms of active step power response. (a) Active and reactive power. (b) Phase A arm current. (c) Capacitance voltage.

can maintain balance under the action of voltage balance control.

The power step test shows that the control effect of the converter station control device is stable, the dynamic performance of the experimental platform is good, and the control targets can respond quickly to the instruction value and can be tracked accurately.

\section{AC Fault Test}

In order to verify the fault test capability of the built platform, examine the ability of the control protection system to handle the fault, and understand the influence of the fault on the system operation, the AC side single-phase to ground fault was carried out.

The AC side fault is set to single-phase grounding of the phase A valve side. After the system is in stable operation, the fault is triggered at $t=3.3 \mathrm{~s}$. The relevant voltage and current waveforms are shown in Fig. 11.

When the phase A single-phase to ground fault occurs on the valve side, the DC current fluctuates with a small amplitude.

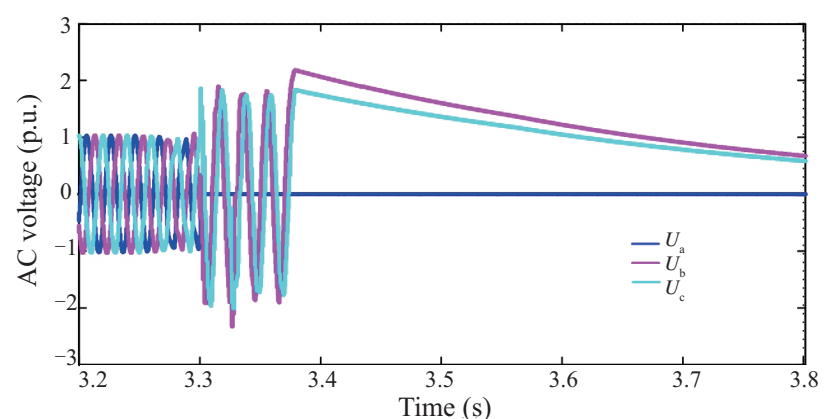

(a)

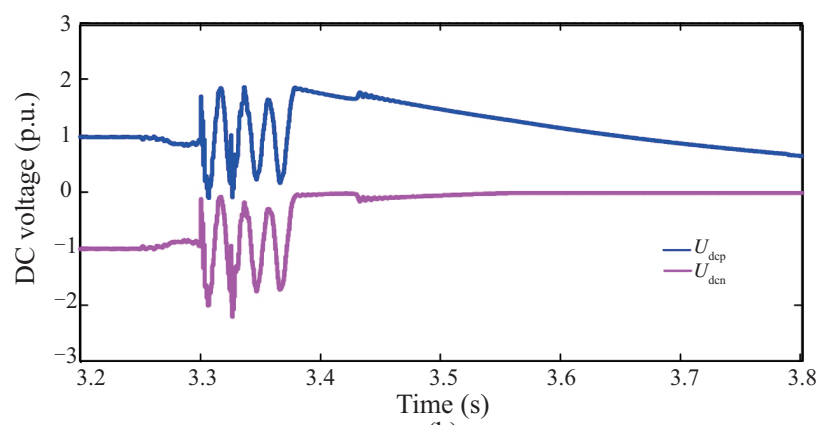

(b)

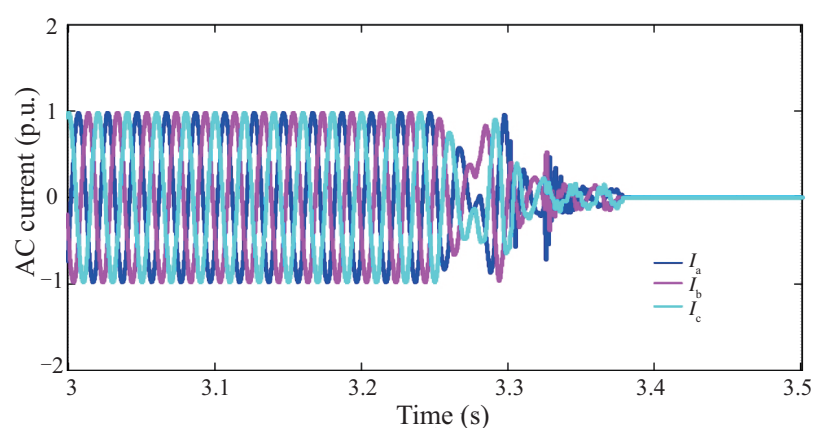

(c)

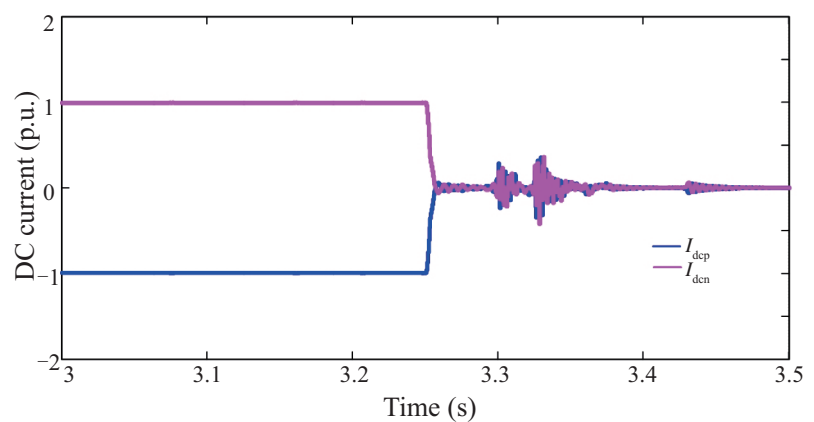

(d)

Fig. 11. Waveforms of valve side single-phase to ground fault. (a) AC voltage. (b) DC voltage. (c) AC current. (d) DC current.

Although the DC voltage appears similar to the sinusoidal fluctuation, the DC voltage can be maintained at $320 \mathrm{kV}$. The three-phase AC current remains basically stable, and the phase A voltage drop on the valve side is 0 while the voltage of $B$ and $\mathrm{C}$ increases to 1.732 times that of the original one. Due to the converter blocking, the discharge path between the submodule capacitance and the short-circuit point is cut off and the AC current is reduced when $t=3.33 \mathrm{~s}$. When $t=3.37 \mathrm{~s}$, the protection occurs and the AC circuit breaker trips. Power 


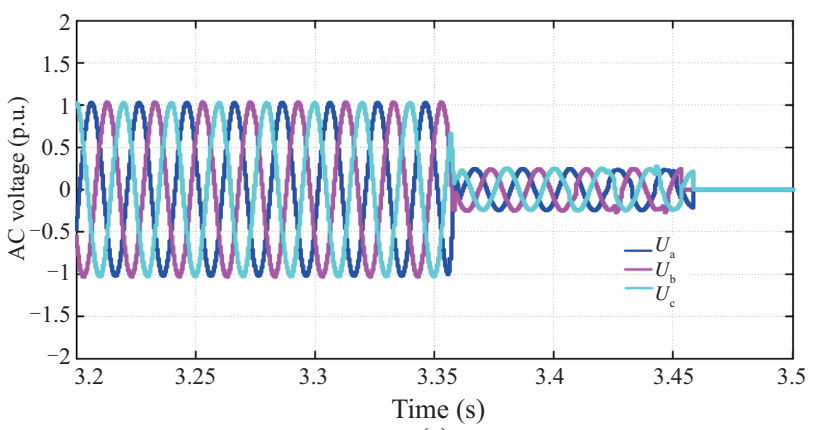

(a)

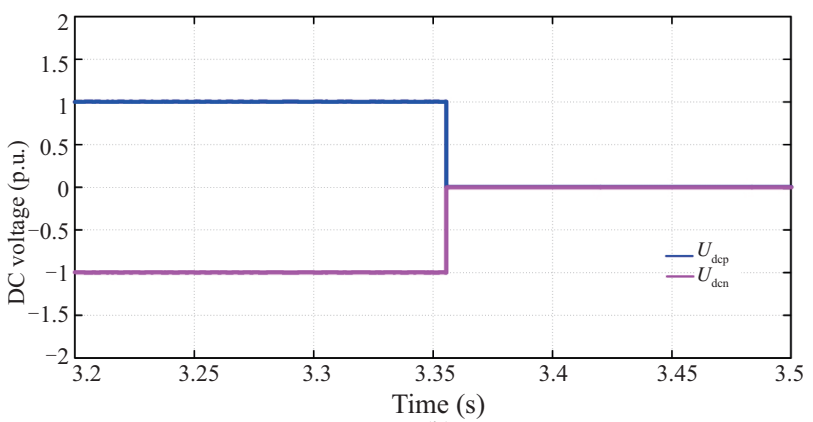

(b)

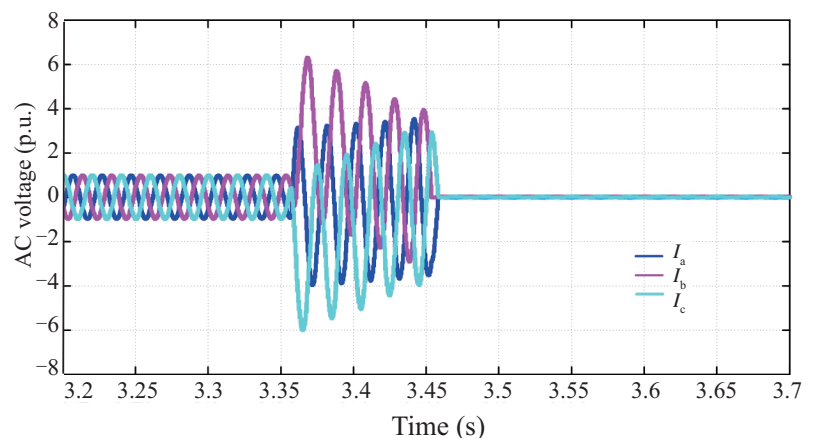

(c)

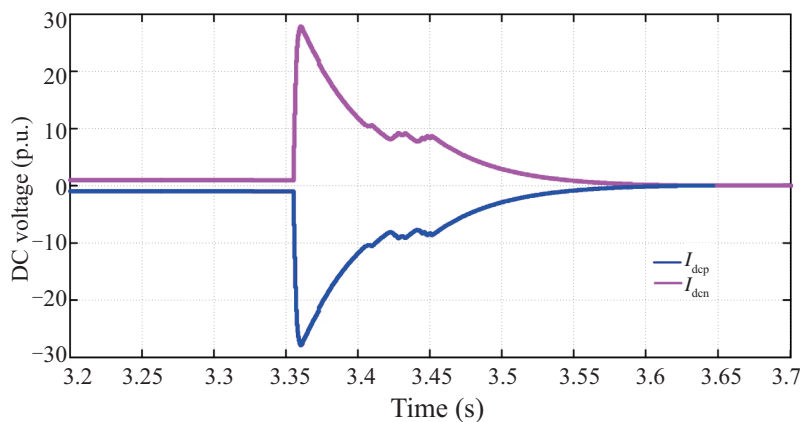

(d)

Fig. 12. Waveforms of valve side single-phase to ground fault. (a) AC voltage. (b) DC voltage. (c) AC current. (d) DC current.

transmission stops and the $\mathrm{AC}$ side can no longer feed current to the $\mathrm{DC}$ side. Therefore, $\mathrm{AC}$ and $\mathrm{DC}$ currents both change to zero.

\section{E. DC Fault Test}

For bipolar short-circuit fault, after the system operates stably, the fault is triggered at $t=3.35 \mathrm{~s}$, the inverter is blocked after $25 \mathrm{~ms}$, and the AC circuit breaker trips at $t=3.45 \mathrm{~s}$. The relevant waveforms obtained from the simulation are given in Fig. 12.

It can be seen from the results in Fig. 12 that after a fault occurs, the capacitance of the submodules in each bridge arm rapidly discharges through the short-circuit path on the DC side, resulting in the rapid reduction of the $\mathrm{DC}$ voltage to 0 , and both the DC current and the bridge arm current will increase rapidly in a short time. With the IGBT blocking, the fault characteristics of the AC side are similar to the three-phase short circuit. It can be seen that the $\mathrm{AC}$ current rises and the $\mathrm{AC}$ voltage decreases. When AC circuit breaker acts, AC current changes to zero. The $\mathrm{DC}$ current is also affected by the inverter blocking and the circuit breaker tripping. Because of the system blocking, the discharge circuit of the bridge arm capacitance is blocked. The DC current no longer contains the capacitor discharge current, and only the $\mathrm{AC}$ side feed current. With the tripping operation of the circuit breaker, the AC system does not feed the current to the DC side anymore, and the DC current gradually decays to zero.

It can be seen from the results in Fig. 12 that after a fault occurs, the capacitance of the sub-modules in each bridge arm rapidly discharges through the short-circuit path on the DC side, resulting in the rapid reduction of the $\mathrm{DC}$ voltage to 0 , and both the DC current and the bridge arm current will increase rapidly in a short time. With the IGBT blocking, the fault characteristics of the $\mathrm{AC}$ side are similar to the three-phase short circuit. It can be seen that the $\mathrm{AC}$ current rises and the $\mathrm{AC}$ voltage decreases. When AC circuit breaker acts, AC current changes to zero. The $\mathrm{DC}$ current is also affected by the inverter blocking and the circuit breaker tripping. Because of the system blocking, the discharge circuit of the bridge arm capacitance is blocked. The DC current no longer contains the capacitor discharge current, and only the $\mathrm{AC}$ side feeds current. With the tripping operation of the circuit breaker, the AC system does not feed the current to the DC side anymore, and the DC current gradually decays to zero.

\section{CONCLUSION}

In this paper, an HIL platform based on RT-LAB for MMC system of two-terminal is built, which has favorable steady-state and dynamic performance. A data merging method between the converter valve control system and the real-time simulator is designed to meet the requirements of communication speed. The test methods of voltage balance control and circulating current suppressing control are proposed. A series of dynamic and fault tests are carried out according to the performance indexes of control and protection devices. The tests show that the proposed test scheme is reasonable and can meet the requirements of the control and protection system test, which can provide an effective means for the study of control strategies. It has certain practical significance for the test of the control and protection device in the flexible DC project and can reduce on-site debugging process and meet its requirements.

\section{REFERENCES}

[1] S. Kouro et al., "Recent advances and industrial applications of multileve converters," in IEEE Transactions on Industrial Electronics, vol. 57, no. 8, pp. 2553-2580, Aug. 2010. 
[2] G. Tang, Z. He, and H. Pang, "Research, application and development of VSC-HVDC engineering technology." Automation of Electric Power Systems, vol. 37, no. 15, 2013, pp. 3-14 (in Chinese).

[3] K. Friedrich, "Modern HVDC PLUS application of VSC in modular multilevel converter topology," 2010 IEEE International Symposium on Industrial Electronics, Bari, 2010, pp. 3807-3810.

[4] N. Flourentzou, V. G. Agelidis, and G. D. Demetriades, "VSCbased HVDC power transmission systems: An overview, " in IEEE Transactions on Power Electronics, vol. 24, no. 3, pp. 592-602, March 2009.

[5] R. Selvamuthukumaran and R. Gupta, "Rapid prototyping of power electronics converters for photovoltaic system application using Xilinx System Generator, " in IET Power Electronics, vol. 7, no. 9, pp. 22692278, September 2014

[6] M. O. Omar Faruque and V. Dinavahi, "Hardware-in-the-loop simulation of power electronic systems using adaptive discretization," in IEEE Transactions on Industrial Electronics, vol. 57, no. 4, pp. 1146-1158, April 2010.

[7] M. Guan and Z. Xu, Fast electro-magnetic transient simulation method for modular multilevel converter, Electric Power Automation Equipment, vol. 32, no. 6, pp. 36-40, 2012.

[8] H. Saad et al., "Dynamic averaged and simplified models for MMCbased HVDC transmission systems, " in IEEE Transactions on Power Delivery, vol. 28, no. 3, pp. 1723-1730, July 2013.

[9] U. N. Gnanarathna, A. M. Gole, and R. P. Jayasinghe, "Efficient modeling of modular multilevel HVDC converters (MMC) on electromagnetic transient simulation programs," in IEEE Transactions on Power Delivery, vol. 26, no. 1, pp. 316-324, Jan. 2011

[10] G. Tang, Z. Xu, and S. Liu, "Improved fast model of the modular multilevel converter," Automation of Electric Power Systems, vol. 38, no. 24, pp. 56-61, 2014.

[11] X. Tu, H. Luo, X. Cheng et al., "Control and protection system interface design for multi-terminal HVDC flexible project," Power System Protection and Control, vol. 43, no. 9, pp. 124-128, 2015.

[12] S. Wu, S. Zhou, J. Qiao et al, "Design of hardware platform test system of HVDC control system," Power System Protection and Control, vol. 42, no. 15, pp. 123-127, 2014

[13] Q. Zhang, Q. Zhao, and P. Chen, "Design of flexible HVDC transmission system simulation platform based on RT-LAB." High Voltage Apparatus, vol. 51, no. 9, pp. 123-128, 2015 (in Chinese).

[14] O. Craciun, A. Florescu, and S. Bacha et al., "Hardware-in-the-loop testing of PV control systems using RT-Lab simulator." in Proceedings of 14th International Power Electronics and Motion Control Conference, EPE-PEMC 2010, 2010, pp. S1-1-S2-6.

[15] S. He, J. Zhang, and X. Cai., "Realization and design of wind power converter model based on RT-LAB HIL system." Power System Protection Control, vol. 41, no. 23, 43-48, 2013.

[16] P. Tian, C. Liu, G. Hong et al., "Design and experimental verification of a hardware-in-the-loop simulation platform for modularized multilevel converter." Automation of Electric Power Systems, vol. 40, no. 1 pp. 122-128, 2016,.

[17] W. Li, J. Chen, M. Chen et al., "RT-LAB real-time simulation research for multi-terminal VSC-DC power distribution network," Guangdong Electric Power, vol. 28, no. 10, pp.78-86, 2015. (in Chinese)

[18] G. Guo, L. Yao and J. Wen, "The group modulation and voltage balance control of the sub-modules in modular multilevel converter." in Proceedings of the CSEE, vol. 36, no. 1, pp. 145-153, 2016. (in Chinese)

[19] M. Guan, Z. Xu, Q. Tu, et al., "Nearest level modulation for modular multilevel converters in HVDC transmission." Automation of Electric Power Systems, vol. 34, no. 2, 2010, pp. 48-52.

[20] Q. Wu, Y. Fu, K Zhang et al., "Research on AC Bus Differential Protection of Flexible DC Transmission System." Power System Protection and Control, vol. 45, no. 3, pp. 100-104, 2017.

[21] P. Qiu, S. Zhang, X. Huang et al., "Research on the protection strategies of internal AC bus fault based on MMC-HVDC." Power System Protection and Control, vol. 42, no. 19, pp. 150-154, 2014.

[22] B Li, Y Li, J He et al., "Stable fault characteristic analysis of the DC system based on modular multilevel converter," Power System Protection and Control, vol. 44, no. 21, pp. 1-8, 2016.

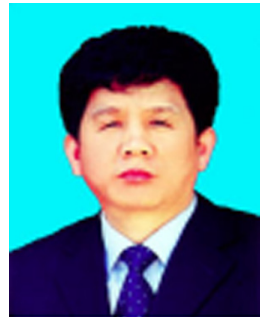

Guoqing Li received M.S. degree in electrical power system and automation from Northeas Electric Power University, Jilin, China, in 1988 , and the $\mathrm{Ph} . \mathrm{D}$. degreein electrical power system and automation from Tianjin University, Tianjin, China, in 1998.

His current research interests include security and stability analysis of power system,flexible HVDC transmission technologyand operation monitoring, protection and control of transmission and distribution equipment

Dr. Li serves as a director of Chinese Society for Electrical Engineering, achairman of electrical mathematics professional committee of Chinese Society for Electrical Engineering, adeputy director of editorial board of Power System Protection and Control, an editorial board of journal of Automation of Electric Power Systemsand a vice chairman of Jilin province Automation Society.

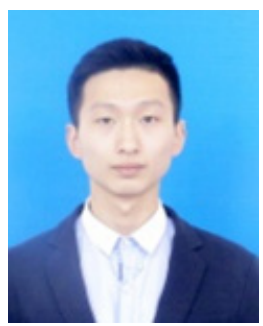

Di Zhang received B.S. degree in electrical engineering and automation from Northeast Electric Power University, Jilin, China, in 2016, where he has been working toward the M.S. degree since 2016

His current research interest isflexible HVDC transmission technology.

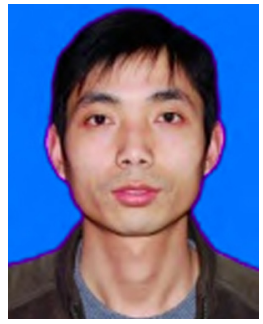

Yechun Xin received M.S. degree in electrical power system and automation from Northeast Electric Power University, Jilin, China, in 2008, and the Ph.D. degreein electrical power system and automation from North China Electric Power University, Beijing, China, in 2015.

His current research interests include flexible HVDC transmission technology,new energy power system,power quality control and energy saving technology and on-line monitoring and fault diagnosis technology of power transmission and distribution equipment.

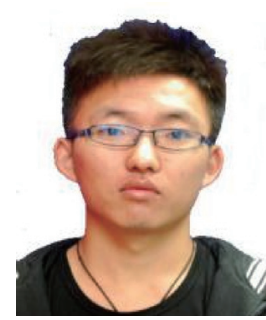

Shouqi Jiang received M.S. degree in electrical engineering and automation from Northeast Electric Power University, Jilin, China, in 2017, where he has been working toward the Ph.D. degree since 2017. His current research interest is flexible HVDC transmission technology.

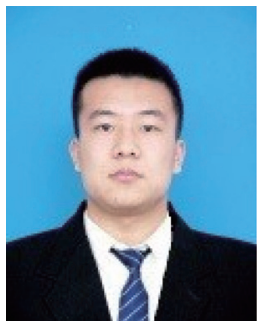

Weiru Wang received M.S. degree in electrical engineering and automation from Northeast Electric Power University, Jilin, China, in 2018, where he has been working toward the Ph.D. degree since 2018. His current research interest is flexible HVDC transmission technology.

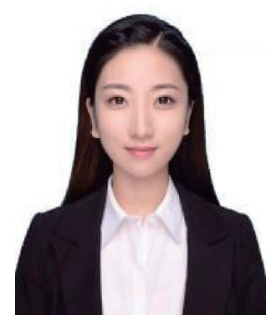

Jiahui Du received B.S. degree in electrical engineering and automation from Northeast Electric Power University, Jilin, China, in 2016, where she has been working toward the M.S. degree since 2016. Her current research interest is power system security and stability. 